\title{
High energy density physics research at IMP, Lanzhou, China
}

\author{
Yongtao Zhao, Rui Cheng, Yuyu Wang, Xianming Zhou, Yu Lei, Yuanbo Sun, Ge Xu, Jieru Ren, Lina Sheng, \\ Zimin Zhang, and Guoqing Xiao \\ Institute of Modern Physics, Chinese Academy of Sciences, Lanzhou 730000, China \\ (Received 30 May 2014; revised 21 October 2014; accepted 4 November 2014)
}

\begin{abstract}
Recent research activities relevant to high energy density physics (HEDP) driven by the heavy ion beam at the Institute of Modern Physics, Chinese Academy of Sciences are presented. Radiography of static objects with the fast extracted high energy carbon ion beam from the Cooling Storage Ring is discussed. Investigation of the low energy heavy ion beam and plasma interaction is reported. With HEDP research as one of the main goals, the project HIAF (High Intensity heavy-ion Accelerator Facility), proposed by the Institute of Modern Physics as the 12th five-year-plan of China, is introduced.
\end{abstract}

Keywords: heavy ion beam; high energy density physics; ion beam and plasma interaction; radiography

\section{Introduction}

High energy density (HED) is generally defined as a state with energy content above $10^{11} \mathrm{~J} \mathrm{~m}^{-3}$, or equivalently with pressure above 1 Mbar. HED states widely exist in the universe, for example the hydrogen in the core of the Sun or in the core of the Jupiter, the iron in the core of the Earth, the water in the core of Uranus, and so on. The creation of such an extreme state in the laboratory and the study of its properties is very challenging and of key importance in the areas of astrophysics, planetary sciences, geophysics, inertial fusion sciences and applications, and so on. In recent years, the primary emphasis in HED research has been given to the physical properties of warm dense matter (WDM), which is a special HED state typically with temperature of the order of $\mathrm{eV}$ and density of the order of solid density, \pm 2 magnitudes. In the WDM region, standard theoretical techniques break down, and experiments are badly needed.

As a potential driver for HED matter or inertial fusion energy (IFE), the heavy ion beam from an accelerator is unique, with the advantages of high repetition rate, large size of sample, homogeneous physical condition, good reproducibility and isometric heating of any target at high density, in addition to the ability to compress matter with a front/side shock at very low entropy. There are also some other important applications of ion accelerators in HED physics (HEDP) research, for example, in studying the atomic processes in plasma, in diagnostics of HED samples

Correspondence to: Y. Zhao, Nanchang Road 509, Lanzhou 730000, China. Email: zhaoyt@impcas.ac.cn by methods of high energy proton/ion radiography and in fast ignition of a compressed fuel. Associated investigations have been pursued with increasing intensity by major accelerator laboratories and institutions in Europe, the USA, Russia, and Japan, where significant progress has been made during the last few decades ${ }^{[1-14]}$.

The Institute of Modern Physics, Chinese Academy of Sciences (IMP) also addresses key issues of HEDP research with the heavy ion beam at the Heavy Ion Research Facility in Lanzhou (HIRFL). In this paper, investigations of radiography by the fast extracted high energy carbon ion beam from the CSR (the Cooling Storage Ring) are introduced, studies on the interaction of a low energy heavy ion beam with plasma are discussed, the project HIAF (High Intensity heavy-ion Accelerator Facility), proposed by the IMP as the 12th five-year-plan of China, and the related parameters and proposals for HEDP research at HIAF are introduced.

\section{Radiography of static objects by the fast extracted high energy carbon beam from the CSR}

In a typical dynamic experiment, diagnostics of the spatial, density and element distribution of a bulk target and their evolution are of key importance. Apart from imaging with self-radiation such as X-rays or neutrons from the target, radiography with a separate bright source is also commonly utilized. Compared with conventional $\mathrm{x}$-radiography, high energy proton or heavy ion radiography is very promising, in particular as it ensures long penetration 

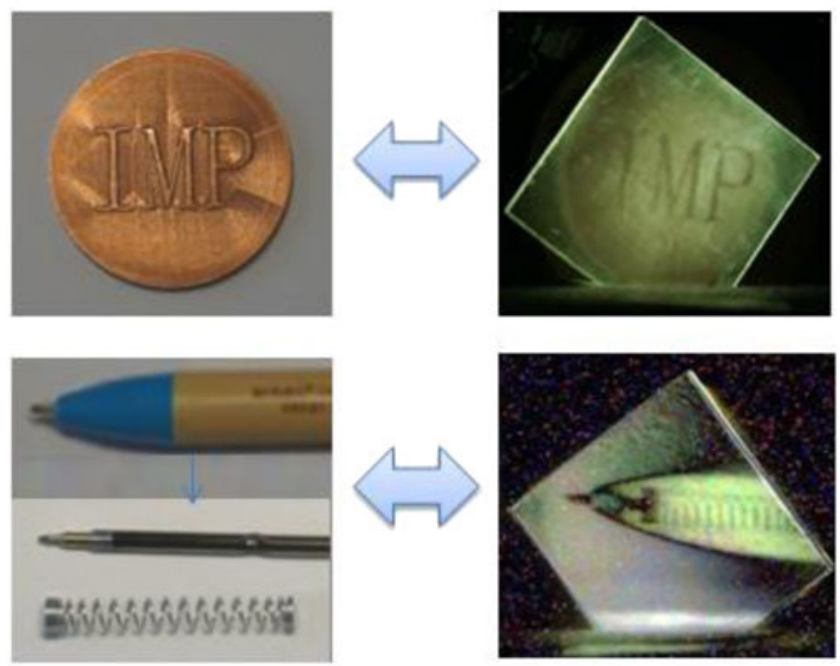

Figure 1. Typical radiographic images (right) of static objects (left) by the method of marginal range radiography.

distance, high spatial resolution, large dynamic range and high sensitivity to the material density. Many successful experiments on high energy proton radiography have been performed in recent decades ${ }^{[15-18]}$. In this section, recent results on high energy carbon beam radiography of static objects are introduced. Methods of both marginal range radiography and magnetic imaging lens radiography have been utilized. The difference of heavy ion radiography from proton radiography will be discussed.

The experiments were performed at the HIRFL, where beams of protons with a maximum energy of $2.8 \mathrm{GeV}$ or carbon with a maximum energy of $1 \mathrm{GeV} / \mathrm{u}$ can be provided. Detailed introduction of the facilities has been reported elsewhere $^{[10]}$.

Figure 1 shows typical radiographic images of an IMP sign and a ballpoint pen, where the marginal range radiography method was used. Approximately $10^{8}$ carbon ions with an energy of $240 \mathrm{MeV} / \mathrm{u}$ were extracted from the CSR at HIRFL in a fast extraction mode, with a pulse duration of about $650 \mathrm{~ns}$. Very good spatial resolution was achieved in the experiment; for example, the diameter of the steel wire inside the ballpoint pen was $400 \mu \mathrm{m}$; the measurement from the radiographic experiment was $389 \pm 21 \mu \mathrm{m}$.

Since the Bragg peak for heavy ions in matter is much sharper, and its transverse distribution at the range margin is much narrower than that for protons, better spatial and density resolution can be expected for heavy ion marginal radiography than for proton marginal radiography (for more details, see our previous report ${ }^{[18]}$ ).

Radiography with magnetic imaging lenses has been tested at the CSR as well. Figure 2 shows the first radiographic image with a $600 \mathrm{MeV} / \mathrm{u}(7.2 \mathrm{GeV}$ in total) carbon beam by utilizing a magnetic imaging lens system with magnifier of around 1:1. The beam intensity is

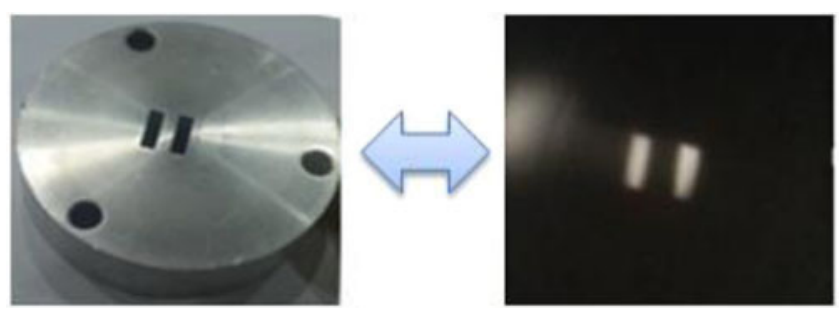

Figure 2. A radiographic image (right) of a static object (left) by the method of magnetic imaging radiography.

approximately $5 \times 10^{9}$ with a pulse duration of about $500 \mathrm{~ns}$. The static object is a piece of $10 \mathrm{~mm}$ thick aluminum plate with two parallel slits; each slit is $10 \mathrm{~mm}$ in length and $3 \mathrm{~mm}$ in width. Since the imaging lenses include two dipole magnets, a bright spot on the left-hand side of the image of the slits was formed by the ions passing through the thick plate. It is calculated that the spatial resolution of the radiographic image was about $65 \mu \mathrm{m}^{[19]}$.

As we know that the principle of magnetic imaging radiography is very similar to transmission electron microscopy (TEM), magnetic imaging lenses in the system can overcome the scattering blur, while a collimator in between the magnets can optimize the contrast. The total momentum and its dispersion, together with the magnifier of the imaging system, may influence the spatial resolution as well. In general, the spatial resolution can be described as following

$$
\Delta x \propto L_{c} \cdot \phi \cdot \frac{\Delta p}{p},
$$

where $L_{c}, \phi$, and $\Delta p / p$ are the chromatic length, the scattering angle, and the momentum dispersion of the beam after transmitting the object, respectively. It is always emphasized that a better resolution could be expected by increasing the momentum (or energy) of the particles, since a higher particle energy may cause less scattering blur, lower momentum dispersion and lower chromatic aberration. By increasing the particle mass (with the same projectile velocity), the same effects (less scattering blur, and so on) can be expected as well; in other words, high energy heavy ion radiography may have a better resolution than proton radiography, if the fragmentation influence and other effects are not taken into account. Moreover, heavy ions always have a much higher energy deposition, so that less beam intensity is required to produce a similarly bright image on the screen, compared with protons.

\section{Interaction of a low energy heavy ion beam with plasma}

Investigation of the interaction processes of ion beams with plasma has attracted a lot of attention during recent decades. The motivations are mainly as follows: (1) the energy deposition process of heavy ions in ionized matter is one of 


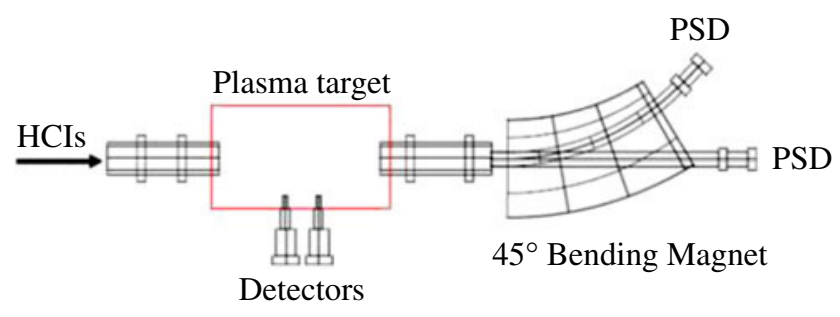

Figure 3. The experimental terminal for studies of low energy ion and plasma interaction at the IMP.

the most important processes in heavy-ion-driven HED and in the burning of inertial confinement fusion (ICF) fuel; (2) plasma devices could serve as important accelerator equipment to focus an ion beam (so-called plasma lens) and/or to strip an ion beam (so-called plasma stripper) ${ }^{[20-26]}$. Apart from these applications, such research is also an important fundamental topic in understanding the atomic processes in plasma, such as the di-electron recombination process, the free electron capture process, the effective charge in the Coulomb interaction process, and so on.

As has been shown in previous experiments, the stopping power of ionized matter is increased compared with that of cold, non-ionized matter. Enhancement factors of the order of 2-3 have been observed at high projectile energies $(E \sim$ $\mathrm{MeV} / \mathrm{u}$ ), depending on the projectile ion species and the free electron density of the plasma ${ }^{[21,22]}$. This effect is especially pronounced at lower ion energies $(E \sim \mathrm{keV} / \mathrm{u})$, where an enhancement factor of up to 35 has been observed ${ }^{[23]}$. Due to the strong nonlinear effects and their special importance in ICF research, more and more emphasis has been given to investigations of ion beams in the low energy range and/or of plasma with high intensity ${ }^{[18,24-26]}$. In this section, the recent progress in research on low energy ion interaction with plasma is briefly introduced.

As shown in Figure 3, an experimental terminal for studies of low energy ion and plasma interaction has been set up on the High Voltage Electronic Cyclotron Resonance ion source (HV-ECR) platform at the IMP. The platform can provide both proton and heavy ion beams with intensity of the order of tens to several thousands of enA, and with energy up to $320^{*} q \mathrm{keV}$ ( $q$ is the charge state of the ions). The energy resolution of the $45^{\circ}$ bending magnet is around $1 \%$, depending on the beam size and the beam divergence.

A gas discharging plasma device, as shown in Figure 4, can generate plasma by igniting an electric discharge in two collinear quartz tubes, and then produce fully ionized hydrogen plasma with a free electron density of $10^{16}-10^{17} \mathrm{~cm}^{-3}$ and with a temperature of $1-2 \mathrm{eV}$. The capacitor bank of about $3 \mu \mathrm{F}$, discharging at a voltage of up to $5 \mathrm{kV}$, produces the electrical current going in two opposite directions in either of the two quartz tubes. Such a design of the plasma target is able to suppress the accelerating effect caused by the electric field between the anode and cathodes. Detailed

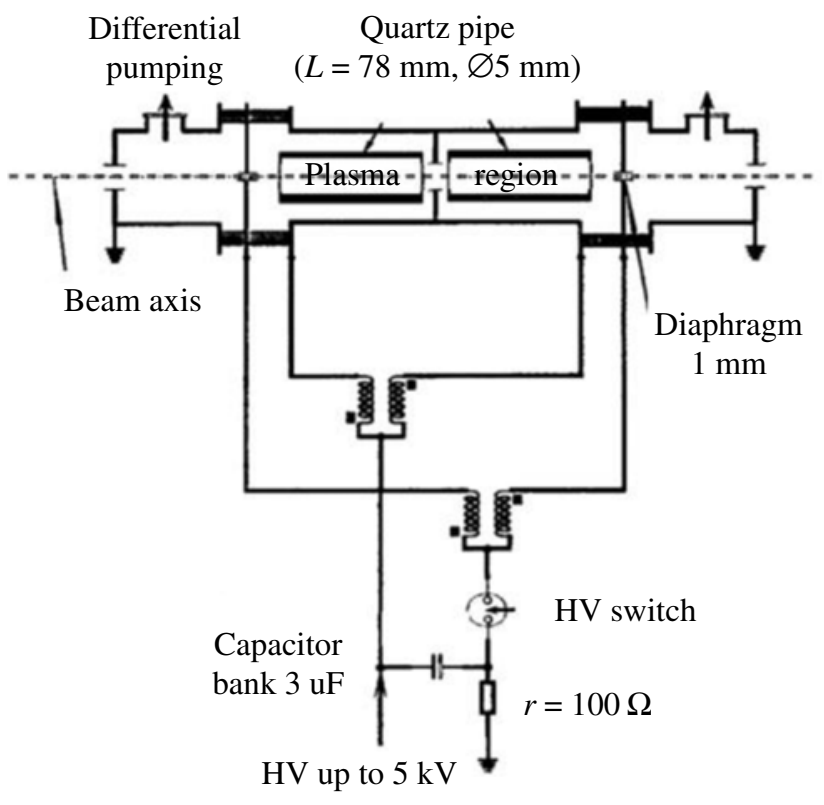

Figure 4. The scheme of the plasma device.

information about the experimental setup and the test results, as well as some simulation results on beam transportation, can be found in previous reports ${ }^{[18,20]}$.

\section{High Intensity heavy-ion Accelerator Facility (HIAF)}

After successful construction of the CSR at HIRFL, a large scale scientific research platform, named the HIAF, was proposed in light of the trend and development in nuclear physics and the associated high energy heavy ion research fields. The proposed platform will be one of the projects for basic sciences and technologies as the 12th five-yearplan in China; it will be a laboratory open to the outside world, similar to CSR which was built as the 9th fiveyear-plan in China. The main goals of this platform are the following: (1) exploration of the effective interactions inside nuclei and the formation of elements heavier than iron in the universe, and other fields related to nuclear physics and nuclear astrophysics; (2) investigation of HEDP and the basic techniques for ICF driven by an intense heavy ion beam; (3) development of the biology and material sciences related to particle irradiation, and so on.

The HIAF complex, as shown in Figure 5, includes a superconductive electronic cyclotron resonance (SECR) ion source, an ion linear accelerator (i-Linac), a booster ring (BRing), a spectrometer ring (SRing), a compression ring (CRing) and about eight experimental terminals; the beam lines or terminals marked in gray in the figure are currently not included in the first stage of the HIAF project. The HIAF will be capable of accelerating ions ranging from proton to uranium, for example, $9.3 \mathrm{GeV}$ protons with an intensity of $1 \times 10^{12}$ ppp (particles per pulse) or $800 \mathrm{MeV} / \mathrm{u} \mathrm{U}^{34+}$ with 


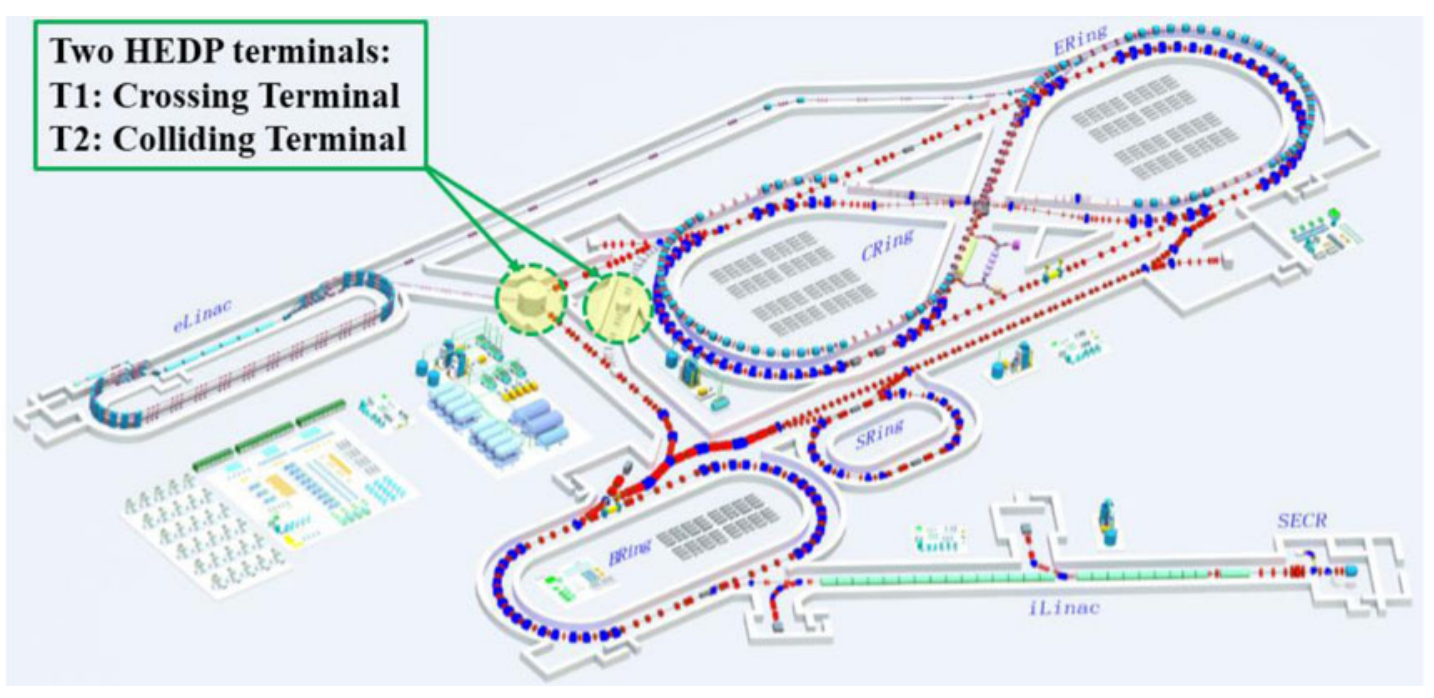

Figure 5. The layout of the HIAF complex and the two terminals for HEDP research.

an intensity of $5 \times 10^{11}$ can be extracted from the BRing, while $12 \mathrm{GeV}$ protons with an intensity of $2 \times 10^{12} \mathrm{ppp}$ or $1.1 \mathrm{GeV} / \mathrm{u} \mathrm{U}^{34+}$ with an intensity of $1 \times 10^{12}$ can be extracted from the CRing.

Since both the BRing and the CRing can produce high energy and high intensity ion beams, two terminals for HEDP research were proposed at the HIAF, one for crossing (T1) and the other for colliding (T2) of the beams from the BRing and the CRing in the target area. Due to budget limitations and technical challenges, only $\mathrm{T} 1$ is included in the first stage of the HIAF project, where HED matter will be produced by the beam from the CRing and diagnosed by the proton radiographic beam from the BRing. High energy electron radiography was proposed to be utilized in the HEDP experiments at the $\operatorname{HIAF}^{[27,28]}$.

Compared with FAIR (Facility for Anti-proton and Ion Research), the power of the final beam for driving a HED sample at the HIAF will be improved due to the advanced design, in particular in the following aspects: (1) the higher energy of the beam from the Linac and the booster ring (serving as injectors) will ensure a higher space charge limit; (2) the larger acceptance of the booster ring will ensure a higher intensity of the injection beam; (3) the powerful electron cooler in the CRing will ensure better focusing and better compression. The key parameters related to HEDP research at the HIAF and other advanced heavy ion drivers are listed in Table 1 , where $E_{0}, N, E_{\text {total }}, S_{f}, t$, and $E_{\rho}$ are the particle energy, the beam intensity (in units of ppp), the total beam energy per pulse, the FWHM of the beam spot, the pulse duration, and the energy density in a lead target, respectively ${ }^{[2-4]}$.

The main topics of HEDP@HIAF will include, for example, the properties of WDM and the related hydrodynamic instabilities, plenary sciences, beam-plasma interaction, fast flyers driven by an intense ion beam, target physics associated with ICF and the related accelerator physics and
Table 1. Key parameters related to HEDP research at the HIAF and other advanced heavy ion drivers (for a uranium beam).

\begin{tabular}{lccc}
\hline & SIS-18 & FAIR (Ph-I) & HIAF (Ph-1) \\
\hline$E_{0}$ & $0.4 \mathrm{GeV} / \mathrm{u}$ & $1 \mathrm{GeV} / \mathrm{u}$ & $1.1 \mathrm{GeV} / \mathrm{u}$ \\
$N$ & $4 \times 10^{9}$ & $4 \times 10^{11}$ & $1 \times 10^{12}$ \\
$E_{\text {total }}$ & $0.06 \mathrm{~kJ}$ & $15 \mathrm{~kJ}$ & $41 \mathrm{~kJ}$ \\
$S_{f}$ & $\sim 1 \mathrm{~mm}$ & $\sim 1 \mathrm{~mm}$ & $1-0.5 \mathrm{~mm}$ \\
$t$ & $130 \mathrm{~ns}$ & $50 \mathrm{~ns}$ & $130-30 \mathrm{~ns}$ \\
$E_{\rho}$ & $2 \times 10^{10} \mathrm{~J} \mathrm{~m}^{-3}$ & $2.4 \times 10^{12} \mathrm{~J} \mathrm{~m}^{-3}$ & $6-24 \times 10^{12} \mathrm{~J} \mathrm{~m}^{-3}$
\end{tabular}

a The upper limit may rely on the budget or upgrading of the HIAF.

technologies. The HIAF is a laboratory open to the outside world; worldwide proposals are welcome.

\section{Acknowledgements}

This work was supported by the Major State Basic Research Development Program of China ('973' Program, grant number 2010CB832902) and the National Natural Science Foundation of China (grant numbers 11105192, 11075192, 11275241, 11375034, and 11275238). We thank D. Hoffmann, R. Piriz, A. Golubev and his colleagues from ITEP, N. Tahir, J. Jocoby, G. Logan, O. Rosmej, Th. Stöhlker, W. L. Zhang and his colleagues from CAEP, X. A. Zhang, $\mathrm{Z}$. Xu, Y. N. Wang, G. Q. Wang and the HIAF committee at $I M P$ for their contributions in the past which have made this work possible.

\section{References}

1. J. Meyer-ter-Vehn, Nucl. Fusion 22, 561 (1986).

2. D. H. H. Hoffmann, V. E. Fortov, M. Kuster, V. Mintsev, B. Y. Sharkov, N. A. Tahir, S. Udrea, D. Varentsov, and K. Weyrich, Astrophys. Space Sci. 322, 167 (2009).

3. N. A. Tahir, Th. Stöhlker, A. Shutov, I. V. Lomonosov, V. E. Fortov, M. French, N. Nettelmann, R. Redmer, A. R. Piriz, C. Deutsch, Y. Zhao, P. Zhang, H. Xu, G. Xiao, and W. Zhan, New J. Phys. 12, 073022 (2010). 
4. N. A. Tahir, C. Deutsch, V. E. Fortov, V. Gryaznov, D. H. H. Hoffmann, M. Kulish, I. V. Lomonosov, V. Mintsev, P. Ni, D. Nikolaev, A. R. Piriz, N. Shilkin, P. Spiller, A. Shutov, M. Temporal, V. Ternovoi, S. Udrea, and D. Varentsov, Phys. Rev. Lett. 95, 035001 (2005).

5. A. A. Golubev and V. B. Mintsev, At. Energy 112, 147 (2012).

6. B. Sharkov, Plasma Phys. Control. Fusion 43, A229 (2001).

7. G. Logan, F. Bieniosek, F. Bieniosek, E. Henestroza, J. Kwan, E. P. Lee, M. Leitner, L. Prost, P. Roy, P. A. Seidl, S. Eylon, J.-L. Vay, W. Waldron, S. Yu, J. Barnard, D. Callahan, R. Cohen, A. Friedman, D. Grote, M. K. Covo, W. R. Meier, A. Molvik, S. Lund, R. Davidson, P. Efthimion, E. Gilson, L. Grisham, I. Kaganovich, H. Qin, E. Startsev, D. Rose, D. Welch, C. Olson, R. Kishek, P. O'Shea, and I. Haber, Nucl. Instrum. Methods A 544, 1 (2005).

8. A. R. Piriz, Y. B. Sun, and N. A. Tahir, Phys. Rev. E 88, 023026 (2013)

9. S. Kawata, Phys. Plasmas 19, 024503 (2013).

10. Y. Zhao, G. Xiao, H. Xu, H. Zhao, J. Xia, G. Jin, X. Ma, Y. Liu, Z. Yang, P. Zhang, Y. Wang, D. Li, H. Zhao, W. Zhan, Z. $\mathrm{Xu}, \mathrm{D}$. Zhao, F. Li, and X. Chen, Nucl. Instrum. Methods B 267, 163 (2009).

11. F. Genco and A. Hassanein, Laser Part. Beams 32, 217 (2014).

12. F. Genco and A. Hassanein, Laser Part. Beams 32, 305 (2014).

13. M. Roth, T. E. Cowan, and M. H. Key, Phys. Rev. Lett. 86, 436 (2001).

14. H. Qin, R. C. Davidson, and B. G. Logan, Phys. Rev. Lett. 104, 254801 (2010).

15. A. M. Koehler and V. W. Steward, Nature 245, 38 (1973).

16. P. A. Rigg, C. L. Schwartz, R. S. Hixson, G. E. Hogan, K. K. Kwiatkowski, F. G. Mariam, M. Marr-Lyon, F. E. Merrill, C. L. Morris, P. Rightly, A. Saunders, and D. Tupa, Phys. Rev. B 77, 220101 (2008).

17. A. A. Golubev, V. S. Demidov, E. V. Demidova, S. V. Dudin, A. V. Kantsyrev, S. A. Kolesnikov, V. B. Mintsev, G. N. Smirnov, V. I. Turtikov, A. V. Utkin, V. E. Fortov, and B. Yu. Sharkov, Tech. Phys. Lett. 36, 177 (2010).
18. Y. Zhao, Z. Hu, R. Cheng, Y. Wang, H. Peng, A. Golubev, X. Zhang, X. Lu, D. Zhang, X. Zhou, X. Wang, G. Xu, J. Ren, Y. Li, Y. Lei, Y. Sun, J. Zhao, T. Wang, Y. Wang, and G. Xiao, Laser Part. Beams 30, 679 (2012).

19. L. Sheng, Y. Zhao, G. Yang, T. Wei, X. Jiang, X. Zhou, R. Cheng, Y. Yan, P. Li, J. Yang, Y. Yuan, J. Xia, and G. Xiao, Laser Part. Beams (2014), doi:101017/S0263034614000676.

20. R. Cheng, Y. Zhao, X. Zhou, Y. Li, Y. Wang, Y. Lei, Y. Sun, X. Wang, Y. Yu, J. Ren, S. Liu, G. Xiao, and D. H. H. Hoffmann, Phys. Scr. T 156, 014074 (2013).

21. K. G. Dietrich, D. H. H. Hoffmann, E. Boggasch, J. Jacoby, H. Wahl, M. Elfers, C. R. Haas, V. P. Dubenkov, and A. A. Golubev, Phys. Rev. Lett. 69, 3623 (1992).

22. D. H. H. Hoffmann, K. Weyrich, H. Wahl, D. Gardés, R. Bimbot, and C. Fleurier, Phys. Rev. A 42, 2313 (1990).

23. J. Jacoby, D. H. H. Hoffmann, W. Laux, R. W. Müller, H. Wahl, K. Weyrich, E. Boggasch, B. Heimrich, C. Stöckl, H. Wetzler, and S. Miyamoto, Phys. Rev. Lett. 74, 1550 (1995).

24. A. Golubev, M. Basko, A. Fertman, A. Kozodaev, N. Mesheryakov, B. Sharkov, A. Vishnevskiy, V. Fortov, M. Kulish, V. Gryaznov, V. Mintsev, E. Golubev, A. Pukhov, V. Smirnov, U. Funk, S. Stoewe, M. Stetter, H.-P. Flierl, D. H. H. Hoffmann, J. Jacoby, and I. Iosilevski, Phys. Rev. E 57, 3363 (1998).

25. A. Frank, A. Blažević, V. Bagnoud, M. M. Basko, M. Börner, W. Cayzac, D. Kraus, T. Heßling, D. H. H. Hoffmann, A. Ortner, A. Otten, A. Pelka, D. Pepler, D. Schumacher, An. Tauschwitz, and M. Roth, Phys. Rev. Lett. 110, 115001 (2013).

26. C. Teske, B. Lee, J. Jacoby, W. Schweizer, and J. C. Sun, Rev. Sci. Instrum. 81, 046101 (2010).

27. F. Merrill, A. Hunt, F. Mariam, K. Morley, C. Morris, A. Saunders, and C. Schwartz, Nucl. Instrum. Methods B 261, 382 (2005).

28. Y. T. Zhao, Z. M. Zhang, and W. Gai, et al. 2013 A high resolution spatial-temporal imaging diagnostic for high energy density physics experiments (submitted). 